\title{
Perfil de saúde bucal dos pacientes internados no Hospital Universitário Maria Aparecida Pedrossian, Campo Grande (MS)
}

\author{
Oral health profile of admitted patients at university hospital Maria Aparecida \\ Pedrossian, Campo Grande (MS) \\ Perfil de la salud oral de los pacientes ingresados en el Hospital Universitario \\ Maria Aparecida Pedrossian, Campo Grande (MS) \\ Deisi Carneiro da COSTA ${ }^{1}$ \\ Karla Ferreira Dias SALDANHA ${ }^{2}$ \\ Albert Schiaveto de SOUSA ${ }^{3}$ \\ Ellen Cristina GAETTI JARDIM
}

\author{
${ }^{1}$ Residência em Odontologia Hospitalar no Hospital Universitário Maria Aparecida Pedrossian, Campo Grande (MS) \\ ${ }^{2}$ Residência em Odontologia Hospitalar no Hospital Universitário Maria Aparecida Pedrossian, Campo Grande (MS) \\ ${ }^{3}$ Professor do Departamento de Morfofisiologia do Centro Ciências Biológicas e da Saúde da \\ Universidade Federal de Mato Grosso do Sul, Campo Grande (MS) \\ ${ }^{4}$ Prof ${ }^{a}$. Adjunto da Disciplina de Cirurgia Bucomaxilofacial da Universidade Federal de Mato Grosso do Sul \\ e Preceptora da Residência em Odontologia Hospitalar no Hospital Universitário Maria Aparecida Pedrossian, Campo Grande (MS)
}

\begin{abstract}
Resumo
A hospitalização está associada a uma piora das condições de saúde bucal sendo assim, o cirurgião dentista no ambiente hospitalar atua na concretização do conceito de saúde integral e promoção da saúde. O objetivo deste estudo é conhecer o perfil epidemiológico bucal dos pacientes internados no Núcleo de Hospital Universitário Maria Aparecida Pedrossian da Universidade Federal do Mato Grosso do Sul (NHU-UFMS). Esta pesquisa foi desenvolvida de forma retrospectiva e aborda uma minuciosa e detalhada análise das informações dispostas no prontuário clínico. Foram incluídos no estudo os pacientes internados no período de março de 2012 a maio de 2013. A amostra envolveu um total de 188 pacientes, com idade média de 55,19 anos. As principais alterações extrabucais evidenciadas foram: assimetria de face (10,11\%), linfadenopatia (5,32\%), lábios desidratados ou descamativos $(38,83 \%)$, lesão ulcerada em lábios $(15,43 \%)$ e queilite angular $(12,77 \%)$. Dentre as alterações intrabucais identificouse principalmente: redução no fluxo salivar $(27,13 \%)$, língua despapilada $(17,55 \%)$, lesões de origem traumática na mucosa bucal (16,49\%), candidíase $(20,21 \%)$ e estomatite protética $(6,38 \%)$. Dentre os pacientes dentados foi relatado: gengivite $(77,24 \%)$, cálculo dental $(46,90 \%)$, mobilidade dental $(21,38 \%)$, cárie dental $(55,86 \%)$, raiz residual $(42,07 \%)$ e necessidade de exodontia $(51,72 \%)$. Quanto à higiene bucal 30,32\% da amostra apresentou uma condição satisfatória, 47,34\% deficiente e 22,34\% classificados com higiene bucal precária. Através deste estudo fica evidente a importância do cirurgião dentista em âmbito hospitalar, seja no tratamento de sequelas ou na prevenção dos fatores complicadores relacionados com a cavidade bucal durante o período da hospitalização.
\end{abstract}

Descritores: Equipe Hospitalar de Odontologia; Higiene Bucal; Saúde Bucal; Manifestações Bucais.

\begin{abstract}
Hospitalization is associated with a worsening of the oral health status and thus the dentist in the environment hospital operates in the implementation of the concept of integral health and health promotion. The aim of this study is to know oral epidemiological profile of patients hospitalized at the University Hospital Center of Maria Aparecida Pedrossian Federal University of Mato Grosso do Sul (NHU-UFMS). This research was conducted retrospectively and addresses a thorough and detailed analysis of the information set forth in the clinical record. The study included patients hospitalized from March 2012 to May 2013. The sample included a total of 188 patients with a mean age of 55.19 years. The main extraoral changes were evident: the face of asymmetry $(10.11 \%)$, lymphadenopathy $(5.32 \%)$ dehydrated or scaly lips $(38.83 \%)$, ulcerated lesion on lips $(15.43 \%)$ and angular cheilitis (12.77\%). Between the intraoral changes identified mainly: reduction in salivary flow (27.13\%), despapilada language $(17.55 \%)$, injuries of traumatic origin in the oral mucosa $(16.49 \%)$, candidiasis $(20.21 \%)$ and denture stomatitis $(6.38 \%)$. Among patients dentate was reported: gingivitis (77.24\%), dental calculus (46.90\%), tooth mobility (21.38\%), dental caries (55.86\%), root residual (42.07\%) and the need for extraction (51.72\%). Regarding oral hygiene $30.32 \%$ of the sample had a condition satisfactory, $47.34 \%$ and $22.34 \%$ poor classified as poor oral hygiene. Through this study it is evident importance of the dentist in the hospital environment, is in the treatment of sequelae or prevention of the factors related complicating the oral cavity during the period of hospitalization.
\end{abstract}

Descriptors: Dental Staff, Hospital; Oral Hygiene; Oral Health; Oral Manifestations.

\section{Resumen}

La hospitalización se asocia con un empeoramiento del estado de salud oral y por lo tanto el dentista en el entorno el hospital opera en la puesta en práctica del concepto de salud integral y la promoción de la salud. El objetivo de este estudio es conocer perfil epidemiológico bucal de los pacientes hospitalizados en el Centro Hospitalario Universitario de Maria Aparecida Pedrossian Universidad Federal de Mato Grosso del Sur (NHU-UFMS). Esta investigación se realizó de forma retrospectiva y direcciones un análisis completo y detallado de la información contenida en la historia clínica. El estudio incluyó a pacientes hospitalizado desde marzo de 2012 hasta mayo de 2013 . La muestra incluyó un total de 188 pacientes con una edad media de 55.19 años. Los principales cambios extraorales eran evidentes: la cara de la asimetría (10,11\%), linfadenopatía (5,32\%) los labios deshidratados o escamosas $(38,83 \%)$, lesión ulcerada en los labios $(15,43 \%)$ y queilitis angular $(12,77 \%)$. entre la cambios intraorales identificaron principalmente: la reducción del flujo salival (27,13\%), el lenguaje despapilada $(17,55 \%)$, lesiones origen de la traumática en la mucosa oral $(16,49 \%)$, la candidiasis $(20.21 \%)$ y estomatitis protésica $(6,38 \%)$. entre los pacientes Se informó dentada: la gingivitis $(77,24 \%)$, cálculo dental $(46,90 \%)$, la movilidad dental $(21,38 \%)$, la caries dental $(55,86 \%)$, raíz residual $(42,07 \%)$ y la necesidad de extracción $(51,72 \%)$. En cuanto a la higiene oral $30,32 \%$ de la muestra tenía una condición satisfactoria, $47.34 \%$ y $22.34 \%$ pobres clasificada como pobre higiene oral. A través de este estudio es evidente importancia del dentista en el entorno de hospital, es en el tratamiento de secuelas o la prevención de los factores relacionadas que complica la cavidad oral durante el período de hospitalización.

Descriptores: Personal de Odontología en Hospital; Higine Bucal; Salud Bucal; Manifestaciones Bucales. 


\section{INTRODUÇÃO}

O cirurgião dentista desempenha papel importante no contexto da equipe hospitalar atuando na concretização do conceito de saúde integral, suporte à equipe nos cuidados assistenciais, dinamização e otimização do trabalho interdisciplinar, participando do processo de tomada de decisões, baseado na sólida compreensão de novas terapias e das condições fisiológicas e psicológicas dos pacientes $^{1,2}$.

Sabe-se que a hospitalização está associada à piora das condições de saúde bucal, principalmente nos pacientes em intubação orotraqueal ${ }^{1}$. Levando a crer que indivíduos hospitalizados necessitam de intervenção odontológica de caráter curativo e preventivo $^{3}$.

O profissional de odontologia quando inserido no ambiente hospitalar atua desde a promoção e prevenção em saúde até o tratamento mais especializado de agravos no sistema estomatognático, revertendo o desenvolvimento de doenças em estágios iniciais, tais como cárie, doença periodontal, alterações na mucosa bucal, disfunção temporamandibular e também no diagnóstico de neoplasia bucal ${ }^{1,2}$. É o profissional responsável por difundir os conhecimentos de higiene bucal para os pacientes, acompanhantes e equipe auxiliar, de forma que esta seja incorporada à rotina hospitalar ${ }^{1,2}$. Essa ação é importante, pois o biofilme dental de pacientes hospitalizados é colonizado por microrganismos mais virulentos que os encontrados naturalmente em indivíduos saudáveis, consequentemente o risco de infecção é elevado ${ }^{1}$.

A higiene bucal não se restringe apenas a uma questão de conforto e bem estar, mas sim a um procedimento capaz de prevenir o desenvolvimento de pneumonia nosocomial por patógenos originados da cavidade bucal. Deste modo, os conhecimentos de saúde bucal adquiridos pelo paciente durante a internação lhe proporcionarão melhor qualidade de vida após a alta hospitalar ${ }^{4}$.

Neste sentido, a necessidade da assistência odontológica aos pacientes hospitalizados se justifica por várias razões. A odontologia pode ser inserida no âmbito hospitalar, por um baixo custo, alta resolutividade de agravos pré-existentes e como uma forma de promoção da saúde, promovendo ao paciente atenção integral $^{2}$.

Para o adequado fornecimento de serviços e recursos na odontologia hospitalar uma tarefa considerada insubstituível é a epidemiologia. Ter conhecimento do perfil dos agravos à saúde é uma responsabilidade da saúde pública. Infelizmente a demanda dos serviços odontológicos é limitada, sendo assim, cabe ao setor público a cobertura integral dos grupos epidemiológico e economicamente carentes ${ }^{5}$.
A realização de estudos epidemiológicos e a utilização dos dados são fundamentais no processo de planejamento das ações de saúde ${ }^{6}$. As informações coletadas nos estudos também auxiliam na investigação dos fatores que influenciam a saúde, avaliação do impacto das ações propostas e estratégias aplicadas e levantamento de hipóteses a serem testadas em estudos analíticos futuros ${ }^{7,8}$.

O cirurgião dentista precisa estar capacitado para solucionar as dificuldades apresentadas na manutenção da saúde bucal e no tratamento de doenças bucais que afetam a saúde geral dos indivíduos hospitalizados ${ }^{1}$. Para o diagnóstico correto e tratamento adequado, é evidente a importância do conhecimento das alterações mais frequentes na cavidade bucal na população estudada. Deve-se considerar a importância dos cuidados odontológicos a pessoas com alterações sistêmicas, pois se trata de um grupo que necessita de ações diferenciadas quanto à promoção de saúde ${ }^{7}$.

$\mathrm{Na}$ odontologia os levantamentos epidemiológicos são necessários tanto para se conhecer a prevalência como a necessidade de tratamentos. Os dados coletados permitem que sejam feitas gestões de ações em saúde, avaliação da eficácia geral dos serviços e assim como comparar prevalências ${ }^{8}$.

A assistência odontológica aos pacientes internados no Hospital Universitário Maria Aparecida Pedrossian da Universidade Federal do Mato Grosso do Sul (NHU-UFMS), é feita através da atuação da odontologia na Equipe da Residência Multiprofissional em Saúde - Atenção ao Paciente crítico desde 2010. No entanto até o momento não foi realizado um levantamento epidemiológico das condições de saúde bucal dos pacientes admitidos. Sendo assim, os objetivos deste trabalho foram conhecer o perfil epidemiológico bucal dos pacientes internados no Núcleo do Hospital Universitário Maria Aparecida Pedrossian da Universidade Federal do Mato Grosso do Sul (NHU-UFMS), em Campo Grande/MS, assim como, caracterizar a amostra de acordo com idade, gênero, unidade de internação, comprometimentos sistêmicos mais frequentes, capacidade de autocuidado e desfecho clínico e a frequência de indivíduos afetados pela doença cárie, doença periodontal, edentulismo e uso de prótese odontológica.

\section{MATERIAL E MÉTODO}

Esta pesquisa se caracteriza como um estudo quantitativo, observacional, descritivo retrospectivo, de corte transversal. Aborda uma minuciosa e detalhada análise das informações dispostas no prontuário clínico, com a verificação da condição 
sistêmica e variações da normalidade dos tecidos do sistema estomatognático. Foi submetido à apreciação do Comitê de Ética em Pesquisa da Universidade Federal de Mato Grosso do Sul - UFMS, sendo aprovado pelo parecer de número: 438.345 .

O estudo foi desenvolvido no NHU-UFMS, em Campo Grande/MS, envolvendo os pacientes que foram atendidos pela Residência Multiprofissional em Saúde - Atenção ao Paciente Crítico, internados no período de março de 2012 a maio de 2013, nas enfermarias da Clínica Médica, Doenças InfectoParasitárias e Clínica Cirúrgica I, além da Unidade de Terapia Intensiva - Adulto, Unidade Coronariana e Recuperação Cardíaca Pós-operatória. Foram incluídos na amostra os pacientes com idade superior a 18 anos sendo excluídos os indivíduos indígenas, de comunidades quilombolas, presidiários e institucionalizados, além daqueles com prontuários com informações incompletas.

Os dados extraídos dos prontuários foram registrados em ficha clínica, abordando os dados sociodemográficos, aspectos clínicos das condições de saúde bucal e sistêmica. As informações analisadas foram: idade, sexo, diagnóstico médico, dados da internação hospitalar, presença de comorbidades ou hábitos nocivos, variações da normalidade da mucosa oral e dentição, uso e necessidade de prótese dentária, qualidade da higiene bucal e necessidade de tratamento odontológico.

Nesta pesquisa a qualidade da higiene bucal foi avaliada de acordo com a presença de biofilme, gengivite, saburra, halitose, restos alimentares, secreção de vias aéreas ou do trato digestivo e sangramentos. Classificando-se a ausência todos esses itens ou a presença de apenas 1 (um) destes como higiene satisfatória; dois ou três itens, higiene deficiente; e quatro ou mais itens, higiene precária.

Todos os dados coletados nesta pesquisa foram armazenados em banco de dados informatizado próprio e analisados posteriormente para definir a frequência relativa de cada evento estudado.

\section{RESULTADOS}

Foram incluídos neste estudo 188 pacientes que estiveram internados no NHU-UFMS durante o período de março de 2012 a maio de 2013. Todos os indivíduos da pesquisa foram avaliados e receberam acompanhamento odontológico durante o período da internação, pela equipe de odontologia da Residência Multiprofissional em Saúde - Atenção ao Paciente Crítico, passando por exames clínicos extra e intrabucais, sendo os dados odontológicos registrados no prontuário em fichas de evolução clínica.

A Tabela 1 apresenta os dados referentes às características da amostra quanto à idade, gênero, unidade de internação hospitalar e os principais comprometimentos sistêmicos.

Tabela 1. Características da amostra

\begin{tabular}{|c|c|c|c|}
\hline Característica & $\begin{array}{c}\mathrm{n} \\
(188)\end{array}$ & $100 \%$ & \\
\hline Idade & & & Média \\
\hline 18-35 anos & 41 & 21,81 & \\
\hline 36-59 anos & 60 & 31,91 & 56,05 \\
\hline 60 anos ou mais & 87 & 46,28 & \\
\hline \multicolumn{4}{|l|}{ Gênero } \\
\hline Masculino & 98 & 52,13 & \\
\hline Feminino & 90 & 47,87 & \\
\hline \multicolumn{4}{|l|}{ Unidade de internação } \\
\hline UTI & 51 & 27,13 & \\
\hline $\mathrm{UCO}$ & 45 & 23,94 & \\
\hline Clínica Médica & 42 & 22,34 & \\
\hline DIP & 32 & 17,02 & \\
\hline Clínica Cirúrgica & 18 & 9,57 & \\
\hline \multicolumn{4}{|l|}{ Comprometimento sistêmico } \\
\hline Doença pulmonar & 60 & 31,91 & \\
\hline Doença cardiovascular & 53 & 28,19 & \\
\hline Doença infecto-parasitária & 51 & 27,13 & \\
\hline Doença neurológica & 47 & 25,00 & \\
\hline Doença renal & 28 & 14,89 & \\
\hline Doença gastrointestinal & 24 & 12,76 & \\
\hline Diabetes melittus & 34 & 18,08 & \\
\hline
\end{tabular}

No momento do atendimento odontológico $42,55 \%$ dos pacientes (80 pacientes) se encontravam em ventilação mecânica invasiva por meio de intubação orotraqueal ou traqueostomia e 55,32\% (104 pacientes) não possuíam condições de autocuidado sendo totalmente dependente da equipe de enfermagem. Dentre os participantes do estudo a frequência de alta hospitalar foi de 64,36\% (121 pacientes) e a taxa de óbitos foi de 35,64\% (67 pacientes).

A análise dos achados da avaliação extrabucal evidenciou: alteração na simetria facial em 10,11\% (19 pacientes) dos pacientes, sendo destes 47,37\% (9 pacientes) relacionados a edema de face devido às condições sistêmicas, $10,53 \% \quad$ (2 pacientes) representavam uma assimetria consequente de um abscesso infeccioso, 10,53\% (2 pacientes) por trauma de face e $31,57 \%$ (6 pacientes) dos casos de assimetria estavam associados a outros motivos, como, sequela de acidente vascular encefálico ou de origem não identificada. Lesões traumáticas em lábios decorrentes da fixação do tubo orotraqueal foram descritas em $32,39 \%$ (23 pacientes) dos pacientes que se encontravam em ventilação mecânica e intubação orotraqueal (71 pacientes). Em 38,30\% (72 pacientes) dos prontuários analisados foi relatado que a avaliação odontológica extrabucal não evidenciou nenhuma alteração. Mais informações relacionadas à avaliação extrabucal são descritas na Tabela 2, assim como as principais alterações em tecidos moles identificadas.

Segundo as descrições dos exames intrabucais registrados em prontuário $21,28 \%$ (40 pacientes) dos pacientes apresentavam a mucosa bucal eritematosa, enquanto que $17,55 \%$ (33 pacientes) possuíam mucosa 
bucal hipocorada. $\mathrm{O}$ registro de mucosa oral desidratada foi observado em $21,28 \%$ (40 pacientes) dos prontuários e em 27,13\% (51 pacientes) identificou-se uma redução no fluxo salivar. Evidenciou-se língua despapilada parcial ou totalmente em 17,55\% (33 pacientes) dos pacientes, língua fissurada em 6,38\% (12 pacientes) e língua pilosa em apenas 5 pacientes $(2,66 \%)$.

Tabela 2. Frequência dos achados observados

\begin{tabular}{|c|c|c|}
\hline Avaliação extraoral & n (188) & $100 \%$ \\
\hline Lábios desidratados ou descamativos & 73 & 38,83 \\
\hline Úlcera em lábios & 29 & 15,43 \\
\hline Queilite angular & 24 & 12,77 \\
\hline Assimetria facial & 19 & 10,11 \\
\hline Linfadenopatia & 10 & 5,32 \\
\hline Trismo & 9 & 4,79 \\
\hline Limitação de abertura & 3 & 1,59 \\
\hline Luxação da ATM & 2 & 1,06 \\
\hline Avaliação intraoral & n (188) & $100 \%$ \\
\hline Candidíase & 38 & 20,21 \\
\hline Lesão traumática & 31 & 16,49 \\
\hline Estomatite protética & 12 & 6,38 \\
\hline Hematomas por trauma & 10 & 5,32 \\
\hline Lesões hiperplásicas & 6 & 3,19 \\
\hline Estomatite herpética & 5 & 2,66 \\
\hline Lesões leucoplásicas & 4 & 2,13 \\
\hline Comunicação buco-sinusal & 2 & 1,06 \\
\hline Leucoplasia pilosa & 2 & 1,06 \\
\hline Míase & 2 & 1,06 \\
\hline Mucosite por terapia antiretroviral & 2 & 1,06 \\
\hline Síndrome da ardência bucal & 2 & 1,06 \\
\hline Amiloidose & 1 & 0,53 \\
\hline Úlcera por lúpus eritematoso sistêmico & 1 & 0,53 \\
\hline Estomatite urêmica & 1 & 0,53 \\
\hline Gengivite ulcerativa necrotizante & 1 & 0,53 \\
\hline Leishmaniose & 1 & 0,53 \\
\hline Mucocele & 1 & 0,53 \\
\hline Paracoccidiodomicose & 1 & 0,53 \\
\hline Sarcoma de kaposi & 1 & 0,53 \\
\hline Varicela & 1 & 0,53 \\
\hline Avaliação da arcada dentária & n (145) & $100 \%$ \\
\hline Gengivite & 112 & 77,24 \\
\hline Cárie dental & 81 & 55,86 \\
\hline Necessidade de exodontia & 75 & 51,72 \\
\hline Cálculo dental & 68 & 46,9 \\
\hline Raiz residual & 61 & 42,07 \\
\hline Mobilidade dental & 31 & 21,38 \\
\hline
\end{tabular}

Lesões de origem traumática na mucosa bucal foram relatadas em 16,49\% dos prontuários (31 pacientes), destes pacientes, 11 apresentavam lesões $(35,48 \%)$ localizadas na língua, $15(48,39 \%)$ lesões em mucosa jugal ou labial e $12(38,71 \%)$ lesões em gengiva ou rebordo gengival além de 10 casos $(5,32 \%)$ de hematomas por trauma.

Foi identificado que $23,94 \%$ dos pacientes (45 pacientes) utilizavam prótese total, parcial removível ou prótese fixa. A taxa de edentulismo dentre os pacientes atendidos pela residência multiprofissional foi de 22,87\% (43 pacientes). Destes pacientes, apenas $18(41,86 \%)$ possuíam prótese total.

A qualidade da higiene bucal dos pacientes internados foi classificada em satisfatória, deficiente e precária e está ilustrada na Figura 1, diferenciando o total da amostra em relação aos grupos de pacientes com diagnóstico de pneumonia hospitalar, independentes e dependentes dos cuidados da enfermagem.

Figura 1. Avaliação da qualidade da higiene bucal para o total da amostra, pacientes com diagnóstico de pneumonia, dependentes e independentes dos cuidados da enfermagem, Campo Grande-MS, 2013

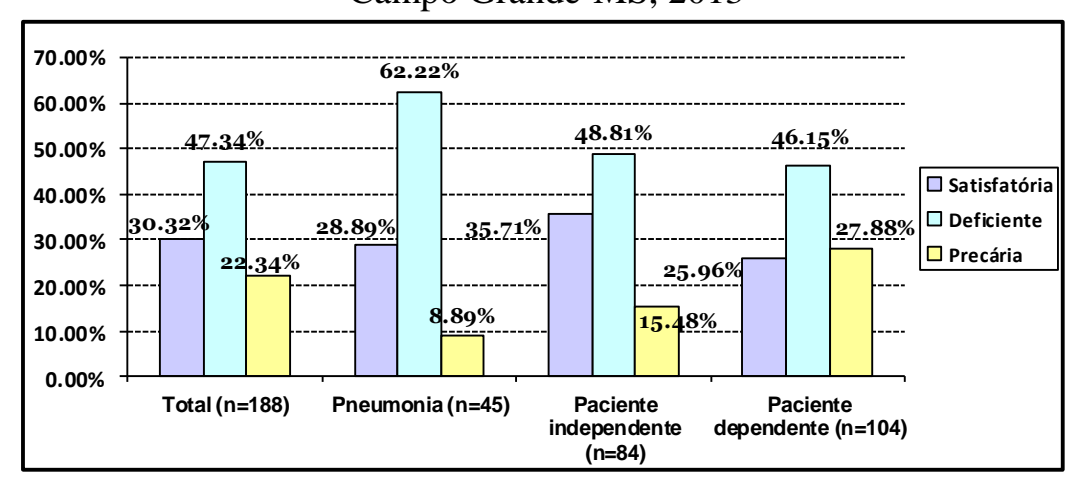

\section{DISCUSSÃO}

O NHU-UFMS está em operação desde 1975, criado com o objetivo fundamental de ser um hospital de ensino, voltado para a formação de recursos humanos na área de saúde. Encontra-se atualmente com 256 leitos, é referência, ao Sistema Único de Saúde no Estado de Mato Grosso do Sul, para atendimento de alta complexidade no tratamento de pacientes com vírus da imunodeficiência adquirida, terapia renal substitutiva e em diagnose, cirurgia cardiovascular, hemodiálise, neurologia, gestação de alto risco, urologia, e tratamento com tomografia e litotripsia além de desenvolver atividades de pesquisa, extensão e servir de campo de estágio para os diversos cursos na área da saúde ${ }^{9}$.

A Residência Multiprofissional em Saúde Atenção ao Paciente Crítico do NHU-UFMS é um programa de residência vinculado ao Ministério da Educação, com duração de 2 (dois) anos teve a sua primeira turma no ano de 2010, composta por 4 (quatro) equipes multiprofissionais contendo cirurgiões dentistas, enfermeiros, farmacêuticos, fisioterapeutas e nutricionistas que em conjunto elaboram um projeto terapêutico singular para cada paciente admitido pela equipe.

Antes do ano de 2010 o NHU-UFMS contava apenas com o serviço e a residência de bucomaxilofacial e com a Faculdade de Odontologia Professor Albino Coimbra Filho da UFMS que quando requerida realizava atendimentos esporádicos nos pacientes internados. Sendo assim a atuação da odontologia de forma efetiva e permanente iniciou-se no NHU-UFMS com o Programa da Residência Multiprofissional no ano 2010, apenas pela ação dos residentes, mesmo sem um profissional da odontologia efetivado para tal assistência. As atividades atuais dos residentes de odontologia incluem avaliação dos pacientes hospitalizados, 
atendimento à beira do leito, atendimento em centro cirúrgico, atendimento em Unidade de Terapia Intensiva, identificação e controle das manifestações orais decorrentes de alterações sistêmicas, assim como o tratamento de condições orais que possam determinar uma complicação sistêmica, além da capacitação e supervisão de equipes auxiliares para a manutenção da saúde bucal.

Os pacientes que foram avaliados pela equipe de odontologia durante o período de março de 2012 a maio de 2013 foram identificados para serem incluídos neste estudo, sendo os dados da internação e as condições da cavidade bucal coletados por meio de análise dos dados registrados em prontuários. A avaliação dos prontuários acabou evidenciando a falta de algumas informações importantes, desta forma, foram efetivamente incluídos neste estudo apenas os pacientes com prontuários contendo todos os dados necessários, totalizando a amostra de 188 pacientes.

A amostra de indivíduos nesta pesquisa é composta por pacientes com idade, gênero, patologias, tempo de internação e capacidade de autocuidado bem distintas, caracterizando-se como uma amostra heterogênea. Ainda assim, pode-se observar uma alta frequência de pacientes idosos $(46,28 \%)$, cerca de metade dos pacientes eram totalmente dependentes dos cuidados da enfermagem (55,32\% - 104 pacientes). $\mathrm{O}$ quadro sistêmico envolvia principalmente doenças pulmonares $(31,91 \%$ - 60 pacientes), cardiovasculares $(28,19 \%$ - 53 pacientes $)$ e infecto-parasitária $(27,13 \%$ - 51 pacientes $)$ e muitos dos pacientes se encontravam em ventilação mecânica (40,43\% - 76 pacientes).

Ainda são escassos na literatura trabalhos que apresentem um panorama das condições bucais de pacientes hospitalizados. Alguns estudos apresentam resultados coletados somente de forma indireta, sem uma avaliação clínica extra e intrabucal realizada por profissional capacitado.

Schneid et al. ${ }^{10}$ registrou em seu estudo as alterações bucais mais frequentemente observadas pela equipe assistencial de enfermagem. Através dos relatos dos profissionais, foi possível identificar condições como halitose (24\%), cáries (33\%), ausência de dentes nos pacientes $(5 \%)$, ulcerações e aftas (33\%), câncer bucal (15\%) e estomatites (5\%). Maestrelli et al. (2010), por meio de aplicação de questionário à pacientes internados, sem executar exame clínico da cavidade bucal, coletou dados quanto às alterações na mucosa oral, como úlceras devido a lesões traumáticas, uso de medicamentos ou uma repercussão do quadro sistêmico $(19,13 \%)$ e o sangramento gengival $(13,04 \%)$. No estudo de Lima et al. ${ }^{11} 64$ pacientes hospitalizados foram interrogados sobre a sua necessidade de tratamento odontológico segundo a sua opinião, foi relatada a necessidade de tratamento periodontal $(67,93 \%)$, exame de rotina $(9,43 \%)$, tratamento ortodôntico $(7,56 \%)$, tratamento restaurador $(3,77 \%)$, troca de prótese $(3,77 \%)$, tratamento endodôntico $(3,77 \%)$ e extração dentária $(3,77 \%)$.

Esta pesquisa não envolveu os indivíduos admitidos pela enfermaria de ortopedia e o NHUUFMS não recebe um grande número de casos de traumatismo, sendo assim as lesões descritas como úlceras ou hematomas de origem traumática frequentemente se tratavam de quadros mordedura involuntária ou ainda estavam associadas à assistência, provocadas, por exemplo, pela intubação orotraqueal, fixação do tubo orotraqueal ou posicionamento de cânulas de Guedel. A frequência de lesões traumáticas em mucosa bucal foi de 16,49\%, localizadas principalmente na mucosa jugal $(48,39 \%)$ além de $5,32 \%$ casos de hematomas intrabucais. Em pacientes sob ventilação mecânica e intubação orotraqueal foi relatado que 32,39\% desenvolveram lesões em lábios relacionadas com a fixação do tubo orotraqueal.

A taxa de edentulismo observada neste estudo foi de 22,87\% (43 pacientes) outros estudos com menor amostra identificaram a frequência de edentados totais variando entre 30 e $39 \%{ }^{12,13}$.

$\mathrm{O}$ estudo realizado por Pires et al. ${ }^{12}$ identificou que indivíduos intubados apresentaram aumento da colonização por Candida spp. de acordo com o tempo de internação, e a saburra lingual mostrou ser o maior reservatório de leveduras na boca. Os microrganismos oportunistas, como Candida spp, podem ser responsáveis pelo desenvolvimento de infecções bucais em pacientes críticos, podendo também levar a pneumonias nosocomiais e lesões bucais ${ }^{12}$. O diagnóstico clinico de candidíase oral realizado pelo cirurgião dentista foi feito em 20,21\% (38 pacientes) dos pacientes avaliados no NHU-UFMS.

Pires et al. ${ }^{12}$ avaliaram a condição oral de pacientes internados em unidade de terapia intensiva $(n=44)$ e observaram úlceras traumáticas $(68,2 \%)$, hematoma de lábio e assoalho da boca (6,8\%), hipossalivação $(84,1 \%)$, acúmulo de biofilme $(90,9 \%)$, saburra lingual $(81,8 \%)$, ressecamento labial $(86,3 \%)$, queilite angular (34\%) e outras lesões associadas à Candida spp. (27,3\%). Os resultados apresentados pelos mesmos autores foram distintos dos encontrados no presente estudo: lesão traumática intraoral 16,49\%, hematoma intraoral por trauma $5,32 \%$, redução do fluxo saliva $27,13 \%$, acúmulo de biofilme 46,28\%, saburra $49,47 \%$, lábios desidratados ou descamativos $38,33 \%$, queilite angular $12,77 \%$ e candidíase oral $20,21 \%$. A presente pesquisa foi realizada em uma amostra maior não sendo restrita à unidade de terapia intensiva, onde se encontram pacientes mais comprometidos sistemicamente e geralmente sob ventilação mecânica o que leva a uma condição bucal 
também mais deficiente, isto pode justificar a diferença entre os resultados encontrados nos dois estudos. Porém ao restringir a amostra e analisar taxa de lesões labiais traumáticas dos pacientes em intubação orotraqueal $(32,39 \%)$ é possível observar semelhança com o resultado apresentado por Pires et al. $^{12}(29,5 \%)$.

As informações registradas nos prontuários revelaram alta frequência de lesões cariosas $(55,86 \%)$, doença periodontal (gengivite 77,24\%, cálculo dental $46,9 \%$, mobilidade dentária $21,38 \%$ ) e presença de raízes residuais $(42,07 \%)$ nos pacientes internados. Esta condição dental representa um foco de infecção para o paciente clinicamente comprometido, risco para complicações sistêmicas, seja pela bacteremia a partir das endotoxinas bacterianas ou pelo estabelecimento de um quadro inflamatório sistêmico crônico de uma infecção localizada na boca apresentando riscos para complicações sistêmicas ${ }^{14,15}$. A condição dentária descrita sugere que muitos indivíduos apresentavam uma condição dental deficiente prévia à internação, sugerindo falta de acesso aos serviços de assistência odontológica. No grupo de pacientes avaliados no estudo de Munro et al. ${ }^{16}$ também foi identificado histórico de problemas dentais anteriores ao período de internação, em média, cada paciente apresentava 9,3 elementos dentários cariados, perdidos ou restaurados.

Alguns pacientes hospitalizados podem apresentar uma condição oral deficiente preexistente à sua internação que é somada a outros fatores que aumentam a colonização bacteriana na cavidade bucal associada à negligência dos cuidados com a saúde bucal durante o processo de hospitalização e às alterações provocadas pela sua condição sistêmica ${ }^{3,16,17}$. Ao longo da internação hospitalar ocorre a deterioração da condição bucal, observa-se um aumento do acúmulo de biofilme dental, da inflamação gengival e alterações na mucosa bucal ${ }^{1,18}$. Neste estudo não foi possível identificar quais as condições bucais foram adquiridas durante a internação das que já eram preexistentes. Podemos considerar os resultados apresentados como uma somatória dos problemas bucais anteriores à internação com os que se desenvolveram durante o processo de hospitalização.

Uma redução no fluxo salivar foi relatada em 27,13\% dos prontuários analisados. Alguns medicamentos interferem no fluxo e $\mathrm{pH}$ salivar, o que influencia diretamente na mucosa oral (xerostomia, alteração sensorial e gustativa, estomatite e hiperplasia gengival) constituindo fatores de risco para doença periodontal e cárie dental ${ }^{3}$.

As condições de saúde do paciente podem também afetar a sua condição bucal, visto que alguns processos patológicos, por exemplo, necessitam de maior ingestão hídrica e, quando esta necessidade não é satisfeita, resulta em halitose, língua saburrosa, presença de cálculo dental, lábios desidratados e fissuras decorrentes do acumulo de microrganismos na boca, podendo exercer um efeito negativo no apetite do paciente, assim como em sua autoestima ${ }^{10}$.

A condição oral do doente pode ser capaz de influenciar no seu estado nutricional que é essencial para a sua recuperação. Pacientes críticos recebem suporte nutricional principalmente pelas via enteral e ou parenteral, no entanto é importante que a alimentação por via oral seja reiniciada o quanto antes. Porém o desconforto e a dor são condições limitantes que podem desencorajar a ingestão de alimentos por via oral ${ }^{19}$. Houve uma alta incidência de alterações dentárias que podem ser consideradas limitantes do ponto de vista funcional do sistema estomatognático como o edentulismo $(22,87 \%)$, falta de reabilitação protética $(41,86 \%$ dos pacientes edêntulos), mobilidade dental por perda de inserção periodontal $(21,38 \%$ dos pacientes dentados), doença cárie $(55,86 \%$ dos pacientes dentados), raízes residuais $(42,07 \%$ dos pacientes dentados) e indicação de exodontias $(51,72 \%$ dos pacientes dentados). Além das lesões identificadas em mucosa bucal que também podem provocar limitações funcionais.

A higiene bucal de indivíduos hospitalizados ou institucionalizados é consideravelmente pior que a de indivíduos da mesma idade não institucionalizados ${ }^{20}$. O NHU-UFMS possui um protocolo de higiene bucal elaborado pela equipe de odontologia da Residência Multiprofissional a ser aplicado pela equipe de enfermagem, porém ainda não há um controle que avalie a frequência e a qualidade da higiene realizada. Neste estudo foi possível evidenciar que a maior parte da amostra apresentava uma higiene bucal deficiente ou precária $(47,34 \%$ e $22,34 \%$ respectivamente). E o grupo de pacientes com independência e capacidade de autocuidado apresentou maior percentual de higiene bucal satisfatória $(35,71 \%)$.

A alta frequência de pacientes apresentando uma higiene bucal deficiente ou precária $(47,34 \%$ e $22,34 \%$ respectivamente) é preocupante, não somente para a condição oral, mas principalmente pelo risco de consequências sistêmicas decorrentes da má qualidade de higiene bucal. A presença do biofilme na cavidade bucal influência a terapêutica médica, devido aos fatores de virulência dos microrganismos que nela se encontram. Os quais podem ser agravados pela presença de outras alterações bucais como a doença periodontal, cáries, necrose pulpar, lesões em mucosas, dentes fraturados ou infectados, traumas provocados por próteses dentárias que podem trazer repercussões na condição sistêmica do paciente ${ }^{21}$.

As atividades educativas e preventivas realizadas pelo cirurgião dentista no meio hospitalar 
são eficientes para melhorar os padrões de higiene bucal de indivíduos hospitalizado sem limitações para a realização do autocuidado ${ }^{22}$. Pacientes de UTIs apresentam higiene bucal deficiente, com quantidade significativamente maior de biofilme do que indivíduos que vivem integrados na sociedade ${ }^{23,24}$, sendo que a quantidade e a complexidade do biofilme bucal aumentam com o tempo de internação ${ }^{23}$.

Espera-se que os resultados obtidos com este estudo possam orientar o planejamento das ações em saúde bucal, visando aperfeiçoar o atendimento odontológico oferecido no NHU-UFMS. A partir desta pesquisa a demanda pelo serviço odontológico pode-se quantificar e assim justificar a necessidade de implantação do serviço de odontologia hospitalar aos pacientes internados no NHU-UFMS.

\section{CONCLUSÃO}

A higiene bucal do paciente hospitalizado foi deficiente ou precária, seja em indivíduos dependentes ou não dos cuidados da equipe de enfermagem. Desta forma, a condição dentária mostrou alta frequência das doenças cárie e periodontal, representando fatores de risco ou focos infecciosos já instalados.

O paciente hospitalizado apresentou um grande número de alterações na cavidade bucal, necessitando de atendimento odontológico durante a internação para a adequada recuperação do seu quadro sistêmico. A presença de lesões traumáticas atuou com um fator complicador para a reabilitação do paciente seja em âmbito local ou mesmo sistêmico.

Através deste estudo fica evidente a importância do cirurgião dentista em âmbito hospitalar, seja no tratamento de sequelas ou na prevenção dos fatores complicadores relacionados com a cavidade bucal durante o período da hospitalização.

\section{REFERÊNCIAS}

1. Terezakis E, Needleman I, Kumar N, Moles D, Agudo E. The impact of hospitalization on oral health: a systematic review. J Clin Periodontol. 2011; 38: 628-36.

2. Mattevi GS, Figueiredo DR, Patrício ZM, Rath IBS. A participação do cirurgião dentista em equipe de saúde multidisciplinar na atenção à saúde da criança no contexto hospitalar. Ciênc saúde coletiva. 2011; 16(10):4229-36.

3. Maestrelli B, Alberton E, Ribeiro DM, CaldoTeixeira AS. Adult patients' profile regarding their oral health conditions and behavior. Int $\mathrm{J}$ Dent. 2010; 9(3):107-13.

4. Araújo RJG, Oliveira LCG, Hanna LMO, Corrêa AM, Carvalho LHV, Alvares NCF. Análise de percepções e ações de cuidados bucais realizados por equipes de enfermagem em unidades de tratamento intensivo. Rev Bras Ter Intensiva 2009; 21(1):38-44.

5. Martins AMEBL, Melo FS, Fernandes FM, Sorte JAB, Coimbra LGA, Batista RC. Levantamentos epidemiológicos brasileiros das condições de saúde bucal. Unimontes Científica 2005; 7(1):5566.

6. Moimaz SAS, Martins CCA, Santos NB, Saliba NA. Aplicação de métodos epidemiológicos nos serviços de saúde bucal da região metropolitana de Belo Horizonte. Arq Odontol 2004; 40(3):221-8.

7. França DCC, Duarte GC, Monteiro AD, Silva AALS, Aguiar SMHA. Perfil epidemiológico dos participantes do Programa de diagnóstico e prevenção do câncer de boca em Mato Grosso. Arq Odontol 2011; 47(2):90-4.

8. Carvalho ES, Bastos RS, Rodrigues ADM, Mello WM, Lauris JRP, Bastos JRM, Peres SHCS. Epidemiologia das doenças bucais em indivíduos na faixa etária entre 35 e 44 anos: o cenário epidemiológico do trabalhador. RGO - Rev Gaúcha Odontol 2010; 58(1):109-14.

9. Fundação Universidade Federal de Mato Grosso do Sul. Hospital Universitário Maria Aparecida Pedrossian. Plano de reestruturação do Hospital Universitário Maria Aparecida Pedrossian HU/UFMS. Campo Grande: Fundação Universidade Federal de Mato Grosso do Sul; 2010.

10. Schneid JL, Berzoini LP, Flores O, Cordon JAP. Práticas de enfermagem na promoção de saúde bucal no hospital do município de Dianópolis-TO. Com. Ciências Saúde 2007; 18(4):297-306.

11. Lima DC, Saliba NA, Garbin AJI, Fernandes LA, Garbin CAS. A importância da saúde bucal na ótica de pacientes hospitalizados. Ciência \& Saúde Coletiva, 16(Supl. 1):1173-1180, 2011.

12. Pires JR, Matareli S, Toledo BEC, Zuza EP. Espécies de Candida e a condição bucal de pacientes internados em unidade de terapia intensiva. Rev Assoc Paul Cir Dent 2011; 65(5):332-7.

13. Caldeira PM, Cabucci RAS. Higiene oral de pacientes em intubação orotraqueal internados em uma unidade de terapia intensiva. Revista Enfermagem Integrada 2011; 4(1) 731-41

14. Feres M, Figueiredo LC. Da infecção focal à medicina periodontal. $\mathrm{R}$ Periodontia 2007;17(2)14-20.

15. Silva JM, Marceliano MFV, Souza PARS, Lamarão SMS. Endodontic infection as factor of risk for systemic manifestations: review of the literature. Rev Odontol UNESP 2007;36(4):357-64.

16. Munro CL, Grap MJ. Oral health and care in the intensive care unit: state of the science. Am J Crit 
Care 2004; 13(1):25-34.

17. Grap MJ, Munro CL, Ashtiani B, Bryant S. Oral care interventions in critical care: Frequency and documentation. Am J Crit Care 2003; 12(2):113-8.

18. Prendergast V, Hallberg IR, Jahnke H, Kleiman C, Hagell P. Oral health, ventilator-associated pneumonia, and intracranial pressure in intubated patients in a neuroscience intensive care unit. Am J Crit Care 2009; 18(4):369-76.

19. Abidia RF. Oral Care in the Intensive Care Unit: A Review. J Contemp Dent Pract 2007; 8(1):76-82.

20. Frasnelli SCT, Oliveira GJPL; Cancian DCJ. O efeito da descontaminação oral na redução dos índices pulmonares nosocomiais-Revisão de literatura. Braz J Periodontol; 21(2):36-44.

21. Rabelo GD, Queiroz CI, Santos PSS. Atendimento odontológico ao paciente em unidade de terapia intensiva. Arq Med Hosp Fac Cienc Med Santa Casa São Paulo. 2010;55(2):67-70.

22. Medeiros Júnior A, Alves MSCF, Nunes JP, Costa ICC. Experiência extramural em hospital público e a promoção da saúde bucal coletiva. Rev Saúde Pública. 2005;39(2):305-10.

23. Morais TMN, Silva A, Avi ALRO, Souza PHR, Knobel E, Camargo LFA. A importância da atuação odontológica em pacientes internados em unidade de terapia intensiva. Rev Bras Ter Intensiva. 2006; 18(4):412-7.

24. Santos PSS, Mello WR, Wakim RCS, Paschoal MAG. Uso de solução bucal com sistema enzimático em pacientes totalmente dependentes de cuidados em unidade de terapia intensiva. Rev Bras Ter Intensiva. 2008;20(2):154-9.

\section{CONFLITO DE INTERESSES}

Os autores declaram não haver conflitos de interesse.

\section{AUTOR PARA CORRESPONDÊNCIA}

Ellen Cristina Gaetti Jardim

ellengaetti@gmail.com
Submetido em 07/03/2016 Aceito em 13/04/2016 